\title{
Novel use of stir bar sorptive extraction (SBSE) as a tool for isolation of oviposition site attractants for gravid Culex quinquefasciatus
}

\author{
C. Carson ${ }^{1 * \dagger}$, M.A. Birkett ${ }^{2}$, J.G. Logan ${ }^{2}$, K. Mawa ${ }^{3}$, \\ H.V. Pates $^{3 \ddagger}$, J.A. Pickett ${ }^{2}$, R.T. Rwegoshora ${ }^{3}$, P.K. Tungu ${ }^{3}$ \\ and M.M. Cameron ${ }^{1}$ \\ ${ }^{1}$ Department of Infectious and Tropical Diseases, London School of \\ Hygiene and Tropical Medicine, Keppel Street, London WC1E 7HT, UK: \\ ${ }^{2}$ Centre for Sustainable Pest and Disease Management, Biological \\ Chemistry Department, Rothamsted Research, Harpenden, UK: ${ }^{3}$ National \\ Institute for Medical Research, Amani Medical Research Centre, Muheza, \\ Tanga, United Republic of Tanzania
}

\begin{abstract}
Mosquitoes such as Culex quinquefasciatus Say (Diptera: Culicidae) are important vectors of organisms that cause disease in humans. Research into the development of effective standardized odour baits for blood-fed females (oviposition attractants), to enable entomological monitoring of vector populations, is hampered by complex protocols for extraction of physiologically active volatile chemicals from natural breeding site water samples, which have produced inconsistent results. Air entrainment and solvent extraction are technically demanding methods and are impractical for use in resource poor environments where mosquito-borne disease is most prevalent. This study reports the first use of a simple, robust extraction technique, stir bar sorptive extraction (SBSE), to extract behaviourally active small lipophilic molecules (SLMs) present in water samples collected from $C x$. quinquefasciatus breeding sites in Tanzania. Extracts from a pit latrine and from a cess pool breeding site attracted more gravid $C x$. quinquefasciatus in pair choice bioassays than control extracts, and coupled gas chromatography-electroantennography (GC-EAG) allowed tentative identification of 15 electrophysiologically active chemicals, including the known oviposition attractant, skatole (3-methylindole). Here, we have demonstrated, using simple pair choice bioassays in controlled laboratory conditions, that SBSE is effective for the extraction of behaviourally and electrophysiologically active semiochemicals from mosquito breeding site waters. Further research is required to confirm that SBSE is an appropriate technique for use in field surveys in the search for oviposition cues for $C x$. quinquefasciatus.
\end{abstract}

*Author for correspondence

Fax: + $44(0) 2476524619$

E-mail: c.carson@warwick.ac.uk

${ }^{\dagger}$ Current address: Populations and Disease Research Group, Department of Biological Sciences, University of Warwick, Gibbet Hill, Coventry, UK

${ }^{\ddagger}$ Current address: Vestergaard Frandsen SA, Chemin de Messidor 5-7, CH-1006 Lausanne, Switzerland 
Keywords: Culex quinquefasciatus, oviposition, SBSE, semiochemical, electroantennography

(Accepted 4 December 2008)

\section{Introduction}

The mosquito Culex quinquefasciatus Say has a widespread geographical distribution and is the vector of many pathogens (causing diseases such as lymphatic filariasis, Japanese encephalitis and West Nile fever) in both tropical and temperate regions (Sucharit et al., 1989; Godsey Jr et al., 2005; Rwegoshora et al., 2005). Gravid Cx. quinquefasciatus females lay clusters of eggs (egg rafts) on the surface of organically polluted standing water (Kirby \& Spence, 1826; Subra, 1981; Becker et al., 2003). Site selection for egg laying is influenced by an oviposition pheromone, (5R,6S)-6-acetoxy-5-hexadecanolide (Laurence \& Pickett, 1985), produced by Culex egg rafts and by other semiochemicals (behaviour and development-modifying chemicals) released from fermenting organic material in the water (Kramer \& Mulla, 1979; Millar et al., 1992). Sampling populations of $C x$. quinquefasciatus involves the use of traps, which exploit this oviposition preference of Culex by releasing semiochemicals to mimic an oviposition site, and hence act as odour baits for the bloodfed gravid female (Surgeoner \& Helson, 1978; Reiter, 1983, 1987; Ritchie, 1984). Natural infusions of organic material, such as grass or hay, with various modifications (Reiter, 1983; Ritchie, 1984; Reisen \& Meyer, 1990; Barbosa et al., 2007; Muturi et al., 2007) are effective as odour baits; however, these formulations lack consistency in their chemical composition. Inherent variability in the organic constituents and the fermentation process means that the attractant properties of such odour baits may vary over time (Kramer \& Mulla, 1979; Isoe et al., 1995a; Sant'ana et al., 2006), leading to inconsistent results in trapping surveys.

To remedy this problem, attempts have been made to develop protocols for the extraction and isolation of volatile semiochemicals from Culex breeding site waters, in order to develop an effective attractant or blend of attractants. Methods, such as air entrainment (Du \& Millar, 1999) or solvent extraction (Millar et al., 1992), have been used to extract small lipophilic molecules (SLMs) from fermented Bermuda grass infusions. When applied to oviposition water, SLMs extracted in this way (such as 3-methylindole (skatole) and 4-methylphenol ( $p$-cresol)) have been shown to function as semiochemicals by attracting significantly more egg laying from gravid females than plain water controls (Millar et al., 1992; Mordue et al., 1992; Beehler et al., 1994). However, whereas the oviposition pheromone is now sold commercially, despite extensive research there is still no commercially available standard odour bait based on a behaviourally active SLM to accompany or synergize pheromone activity. Future studies which aim to address this issue are hampered by the complexity and labour intensive nature of current extraction protocols for breeding site waters, particularly in resource poor environments where mosquito-borne diseases are most prevalent and laboratory facilities are often limited. It is, therefore, a current research priority to identify technically straightforward novel methods for extraction and isolation of potential oviposition attractants in breeding site waters in the field, to be used in the development of a standardized odour bait. A simple, robust analytical technique known as stir bar sorptive extraction (SBSE) has been developed for the collection of SLMs in aqueous media (Baltussen et al., 1999). SBSE has so far been used for purposes such as analysis of water quality (Serodio \& Nogueira, 2005) and clinical samples (e.g. human urine (Kawaguchi et al., 2004)). The technique involves a magnetized bar ('Twister ${ }^{\mathrm{TM}}$; GERSTEL, Anatune, UK), which is coated in polydimethylsiloxane polymer. The stir-bar removes SLMs from a liquid and traps ('sorbs') them temporarily within its matrix for later extraction, either by thermal desorption or by a simple elution step using a high purity solvent such as diethyl ether. Thus, the purpose of the present study was to test the effectiveness of the SBSE technique as a tool for the extraction of putative volatile oviposition semiochemicals from mosquito breeding site water, specifically for Cx. quinquefasciatus in Tanzania, East Africa, for subsequent characterization by GC-EAG and GC-MS.

\section{Materials and methods \\ Ethical approval}

Ethical approval for field work in Tanzania was granted by the Research Ethics Committee at the London School of Hygiene and Tropical Medicine and the National Institute of Medical Research (NIMR), Tanzania.

\section{Survey and identification of breeding sites in Tanzania}

Field work was conducted at Amani Medical Research Centre, Muheza, Tanzania, E. Africa $\left(5^{\circ} 10^{\prime} \mathrm{S}, 38^{\circ} 46^{\prime} \mathrm{E}\right)$ during June-August 2006. A preliminary survey was conducted in and around Muheza to locate aquatic breeding sites for $C x$. quinquefasciatus, confirmed by dipping and identification of larvae and adult specimens. The sites chosen for the study were a domestic pit latrine in Muheza town, and a cess pool at Teule hospital (hereafter referred to in the text as Teule).

\section{Stir bar sorptive extraction of breeding site water}

Samples of breeding site water were sieved through $0.5 \mathrm{~mm}$ mesh to remove organic debris; $250 \mathrm{ml}$ (a volume selected based on previous unpublished work by two of the present authors (M.A.B \& J.G.L.)) was placed in a $300 \mathrm{ml}$ Pyrex beaker and was subjected to extraction using a $20 \mathrm{~mm}$ stir-bar for three hours, using a magnetic stirrer set at $1100 \mathrm{rpm}$ to produce a strong vortex in the liquid.

After extraction, the stir-bar was washed briefly in deionized water, patted dry on absorbent paper and placed into a glass Pasteur pipette. Redistilled HPLC grade diethyl ether $(750 \mu l)$ at room temperature was run through the pipette to elute SLMs from the polymer matrix. The SBSE procedure was performed six times with a fresh $250 \mathrm{ml}$ aliquot of water on each occasion, to generate sufficient volume of sample to conduct subsequent analysis. All six extracts were pooled and re-aliquoted into separate $1 \mathrm{ml}$ glass vials with polytetrafluorethylene (PTFE) seals for storage at $-20^{\circ} \mathrm{C}$ until required. Evaporation of diethyl 
ether that occurred during the pooling procedure reduced the total volume of the samples by around one third, leaving $3000 \mu \mathrm{l}$ extract from $6 \times 250 \mathrm{ml}$ water. Thus, $200 \mu \mathrm{l}$ of the final ether extract contained the volatiles extracted from approximately $100 \mathrm{ml}$ breeding site water.

\section{Bioassays of oviposition activity in response to natural breeding site water}

These experiments aimed to determine whether caged gravid females of $C x$. quinquefasciatus displayed an oviposition preference for natural breeding site water in comparison with clean dechlorinated tap water. Seventy-two hours prior to bioassays, female mosquitoes (3-7 days old) were provided with a bloodmeal using a live laboratory rabbit. Pair choice bioassays were conducted in nylon mesh cages of dimensions $50 \times 50 \times 30 \mathrm{~cm}^{3}$, using standard procedures (Laurence \& Pickett, 1985). Clean plastic tubs $(15 \mathrm{~cm} \varnothing$, $250 \mathrm{ml}$ capacity) were placed side by side, two in each cage, $20 \mathrm{~cm}$ apart. The 'test' tub was filled with $100 \mathrm{ml}$ breeding site water. The 'control' tub was filled with $100 \mathrm{ml}$ clean dechlorinated tap water. The position of test and control tubs was alternated between replicates to control for position effects. Bioassays on pit latrine water were conducted using the MASIMBANI laboratory strain of $C x$. quinquefasciatus, maintained in the insectary at Amani Medical Research Centre. Bioassays on Teule water were conducted using $C x$. quinquefasciatus adult females which originated from this site; pupae had been collected from Teule hospital cess pool by dipping and reared to adult for use in bioassays. All mosquitoes were maintained at $26^{\circ} \mathrm{C}\left( \pm 1^{\circ} \mathrm{C}\right)$ and $80 \%$ $\mathrm{RH}$ in a $12: 12 \mathrm{~h}$ day: night cycle. Each bioassay was run from 18:00h until 08:00 h, and results were recorded by counting the number of egg rafts deposited in test and control tubs. Six replicate tests were performed per bioassay, each using 50 gravid female mosquitoes. Previously untested mosquitoes were used for each replicate.

\section{Bioassays of oviposition activity in response to SBSE extracts}

These experiments, aimed to determine whether caged gravid females of $C x$. quinquefasciatus, displayed a preference for oviposition in water-filled tubs baited with SBSE extracts of breeding site water in HPLC diethyl ether, in comparison with those baited with HPLC diethyl ether only. Bioassays were conducted using $200 \mu \mathrm{l}$ of SBSE extract, equivalent to $100 \mathrm{ml}$ breeding site water. The extract was pipetted onto a glass microscope coverslip using a capillary tube. The coverslip was floated with the extract uppermost, in a clean oviposition tub filled with $100 \mathrm{ml}$ of dechlorinated tap water, supported by the surface tension of the water. The control was a coverslip treated with $200 \mu$ l diethyl ether and floated on $100 \mathrm{ml}$ dechlorinated tap water. All other bioassay conditions were as described above.

\section{Analysis of SBSE extracts by coupled gas chromatography - electroantennography (GC-EAG)}

Extracts were transported to Rothamsted Research (Harpenden, UK) in $1 \mathrm{ml}$ glass vials with PTFE seals. On arrival, samples were concentrated $(\times 5)$ by evaporation of diethyl ether under a gentle stream of nitrogen. To locate electrophysiologically-active compounds within the pit latrine extract, coupled GC-EAG was used as described in previous research (Logan et al., 2008). Briefly, individual gravid females of $C x$. quinquefasciatus MUHEZA (blood fed $72 \mathrm{~h}$ previously, from a colony maintained at LSHTM since 1985) were cooled on ice for $30 \mathrm{~s}$ before removing the head and the tips of both antennae for insertion of indifferent and recording electrodes, respectively. Preparations were held in a continuous, humidified and charcoal-filtered air stream. EAG-active compounds were located by replicating the GC-EAG experiments $(n=6)$ and consistent responses were recorded. An HP 6890 GC was coupled to the electrophysiology set-up to separate components of extracts on a polydimethylsiloxane (HP1) column. Details of oven temperature setup were as described previously.

\section{Gas chromatography (GC) and coupled gas chromatography-mass spectrometry (GC-MS)}

To quantify the EAG-active compounds located within the pit latrine extract, an HP 6890 GC fitted with a non-polar polydimethylsiloxane (HP1) cross-linked capillary column and a polar DB-WAX column was used. For tentative identification of EAG-active compounds, an HP 6890 GC fitted with an HP1 column was coupled to a VG Autospec double-focusing magnetic sector mass spectrometer (Fisons Instruments, Manchester, UK) equipped with an integrated data system (MassLynx V. 4.0). Setup and experimental procedures using this equipment were performed as described by Logan et al. (2008). Compounds were tentatively identified by comparison of mass spectra with those found in the NIST Standard Reference Database (Linstrom \& Mallard, 2007). The identity of EAG-active compounds located in the pit latrine sample was confirmed by peak enhancement on GC using authentic samples of chemicals purchased from a commercial supplier (Sigma-Aldrich, Gillingham, UK).

\section{Statistical analysis}

Data from egg raft counts were $\log _{\mathrm{e}}(n+1)$ transformed before analysis by paired $t$-tests. All statistical analysis was performed using STATA v9.0 (Statacorp, USA).

\section{Results and discussion}

\section{Results of bioassays}

The mean percentage of total egg rafts in test and control oviposition tubs from each bioassay are displayed in fig. 1. Gravid Cx. quinquefasciatus MASIMBANI laid more egg rafts in pit latrine water than in dechlorinated tap water $(t=8.38$; $P<0.001 ; n=6)$, and gravid Teule $C x$. quinquefasciatus mosquitoes laid more egg rafts in Teule water than in dechlorinated tap water $(t=57.60 ; P<0.001 ; n=6)$. Tubs filled with dechlorinated water baited with SBSE pit latrine extract had more egg rafts than water-filled tubs baited with diethyl ether control $(t=10.81 ; P<0.001 ; n=6)$, and Teule mosquitoes responded to Teule SBSE extract by laying higher numbers of egg rafts in comparison with the diethyl ether control $(t=10.19 ; P<0.001 ; n=6)$. These results show that SBSE extracts from Teule hospital cess pool and from the pit latrine site are more attractive to gravid $C x$. quinquefasciatus than diethyl ether controls. The positive effect of these extracts demonstrates that SBSE is effective for extraction of behaviourally active oviposition semiochemicals from 


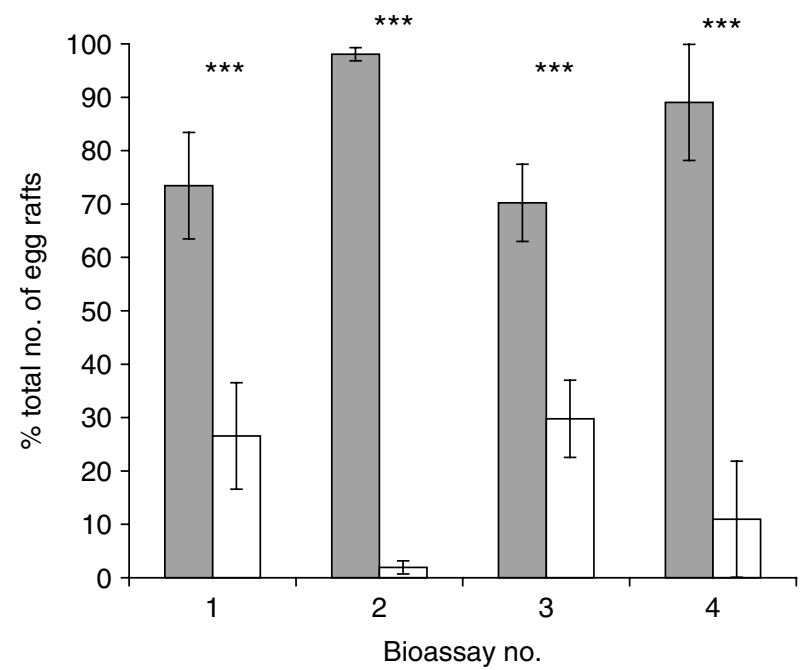

Fig. 1. Mean percentage of total egg rafts (with $95 \%$ confidence interval) in test and control tubs in bioassays of natural breeding site water and SBSE extracts from pit latrine and Teule hospital cess pool versus dechlorinated tap water controls (either with or without HPLC ether, as appropriate). 1, Pit latrine water vs. clean dechlorinated water; 2 , Teule water vs. clean dechlorinated water; 3, Pit latrine extract in HPLC ether on clean dechlorinated water vs. control HPLC ether on clean dechlorinated water; 4, Teule extract in HPLC ether on clean dechlorinated water vs. control HPLC ether on clean dechlorinated water ( $\square$, test tub; $\square$, control tub; $\left.{ }^{* * *}, P<0.001\right)$.

mosquito breeding site water. To our knowledge, this study is the first to demonstrate successfully the use of SBSE for this purpose.

\section{Results of analysis of breeding site water SBSE extracts by GC-EAG and GC-MS}

Coupled GC-EAG analysis located 15 compounds in the behaviourally active pit latrine SBSE extract which elicited EAG activity via the olfactory receptor neurones of gravid female $C x$. quinquefasciatus. A GC-EAG trace for the pit latrine SBSE extract is shown in fig. 2. Identification of these compounds by coupled GC-MS and calculated levels present in the original breeding site waters are shown in table 1. Six of the chemicals detected in SBSE extracts were also present in the control HPLC diethyl ether sample (3-phenylbutan-2ol, butyl methyl ether, heptane, 1,2-dimethylcyclopentane and two unidentified chemicals with R.I values of 890 and 801 , respectively) and were considered likely to be contaminants. The identity of seven of the latrine-specific EAG-active compounds shown in table 1 was confirmed by GC peak enhancement using authentic samples of chemicals.

Successful extraction and subsequent GC-EAG/GC-MS identification of the known oviposition attractant 3-methylindole (skatole) (Millar et al., 1992; Mordue et al., 1992; Beehler et al., 1994), an expected component of faecally contaminated waters such as those collected, provided a biologically plausible basis for attractant properties of these extracts in pair choice bioassays. The amount of skatole present in the behaviourally active pit latrine and Teule SBSE extracts corresponded to a concentration of $0.00458 \mu \mathrm{gl}^{-1}$ and $0.01602 \mu \mathrm{gl}^{-1}$, respectively, in the original breeding site water. These levels are much higher than the skatole concentration of $10^{-4}-10^{-5} \mu \mathrm{gl}^{-1}$, which was reported in previous research to elicit maximal oviposition activity (Blackwell et al., 1993; Olagbemiro et al., 2004), but much lower than that which was reported to cause a repellent effect $\left(10 \mu \mathrm{gl}^{-1}\right)$ (Millar et al., 1994). Interestingly, the oviposition pheromone $(5 R, 6 S)$-6-acetoxy-5-hexadecanolide was not detected in GC-MS analysis of extracts, which may indicate either that it was not present in water samples (perhaps since it evaporates directly into the atmosphere from the apical droplet of the egg) or that it was not extracted with other SLMs in the breeding site water by the SBSE procedure, a possibility that warrants further research. Loss of highly volatile fractions, by evaporation during processing of extracts or whilst performing concentration under nitrogen, is also possible. Other compounds detected in SBSE extracts were not of natural origin and may represent man-made contaminants, for example 1,3,5 trimethylbenzene, which is a product of petroleum contamination. In a previous study, a range of benzene derivatives were isolated from headspace odours of worn stockings, which were attractive to hostseeking females of Anopheles gambiae (Qiu, 2005). However, this class of synthetic chemicals may have been released from the nylon material itself, rather than forming a component of host odour. Phthalates could possibly originate from plasticizers used with PVC, whereas 1-methylnaphthalene can be derived from fossil fuels, such as petroleum and coal, or by pyrolysis of botanical material. The sources of these unexpected components of pit latrine and Teule cess pool extracts are unknown; for future research utilizing the SBSE procedure, it is important to distinguish between contaminants present in the original field breeding site water sample (e.g. due to disposal of automotive oil, cooking fuel (kerosene) or partially combusted organic matter thrown into latrines) and those present due to the sampling methodology (e.g. contaminants in ether or use of plastic receptacles for water collection). Such distinction is necessary because gravid mosquitoes may have adapted their oviposition behaviour to respond to non-natural cues in contaminated breeding site water through conditioning during their immature stages (McCall \& Eaton, 2001). Thus, the search for oviposition attractants for $C x$. quinquefasciatus should not necessarily be restricted to substances of natural origin.

Although this study represents an important step towards validation of a technique for the future isolation of attractants for use as gravid trap odour baits, it is necessary to be cautious in the interpretation of findings based on egg raft counting in laboratory pair choice bioassays. This methodology has been widely used to investigate the attractant properties of different chemicals for gravid female mosquitoes, but some investigators have questioned its validity (Isoe et al., 1995b; Du \& Millar, 1999). A behaviourally active semiochemical may function as an attractant (which induces gravid females to make oriented movements towards its source), an arrestant (which induces turning or hovering in the vicinity) or an oviposition stimulant (which promotes the act of oviposition) (Dethier et al., 1960). Bioassays which use counting of egg rafts as a measure of 'attractiveness' of an oviposition site are measuring the summation of all of the above effects. It, therefore, is not possible to state whether the SBSE extracts in this study functioned as long range attractants, short range oviposition stimulants or a combination of the two. The results of simple pair 


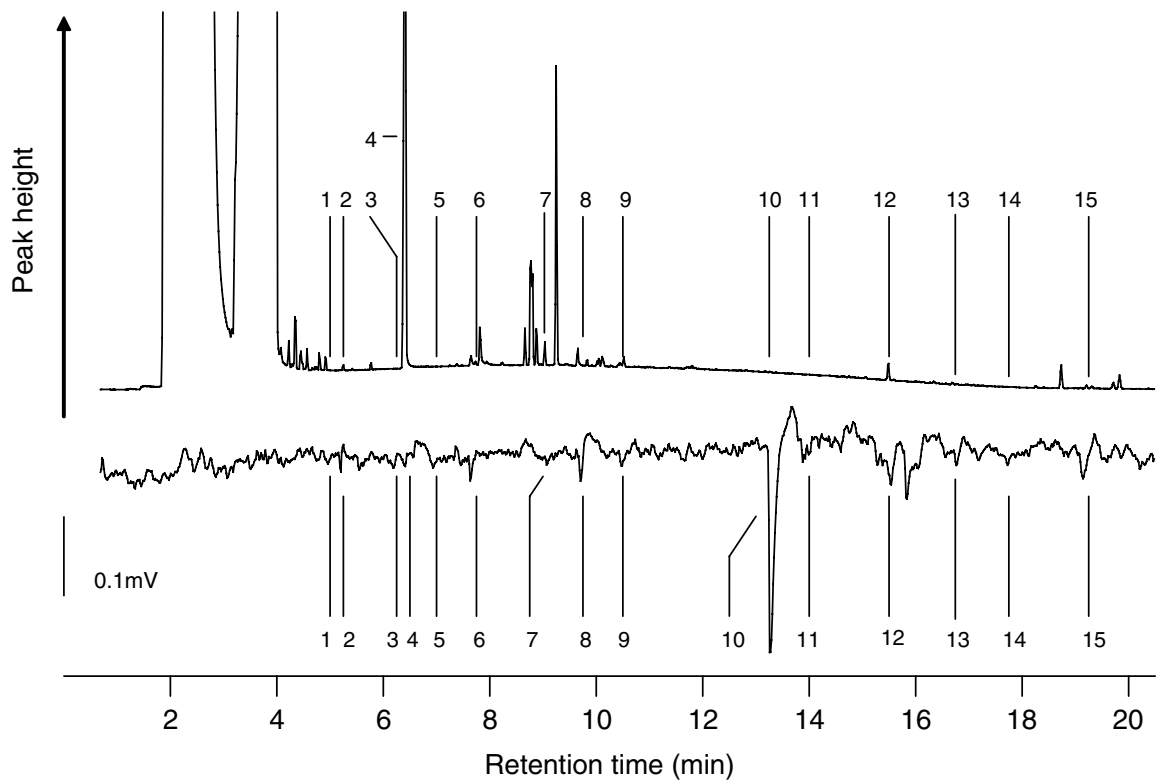

Fig. 2. Results of coupled GC-EAG on pit latrine SBSE extract. The upper trace corresponds to the FID detector on the GC. The lower trace corresponds to the antennal response of the insect preparation. Numbered peaks correspond to the compounds listed in table 1.

Table 1. Identification of electrophysiologically active fractions of SBSE extracts.

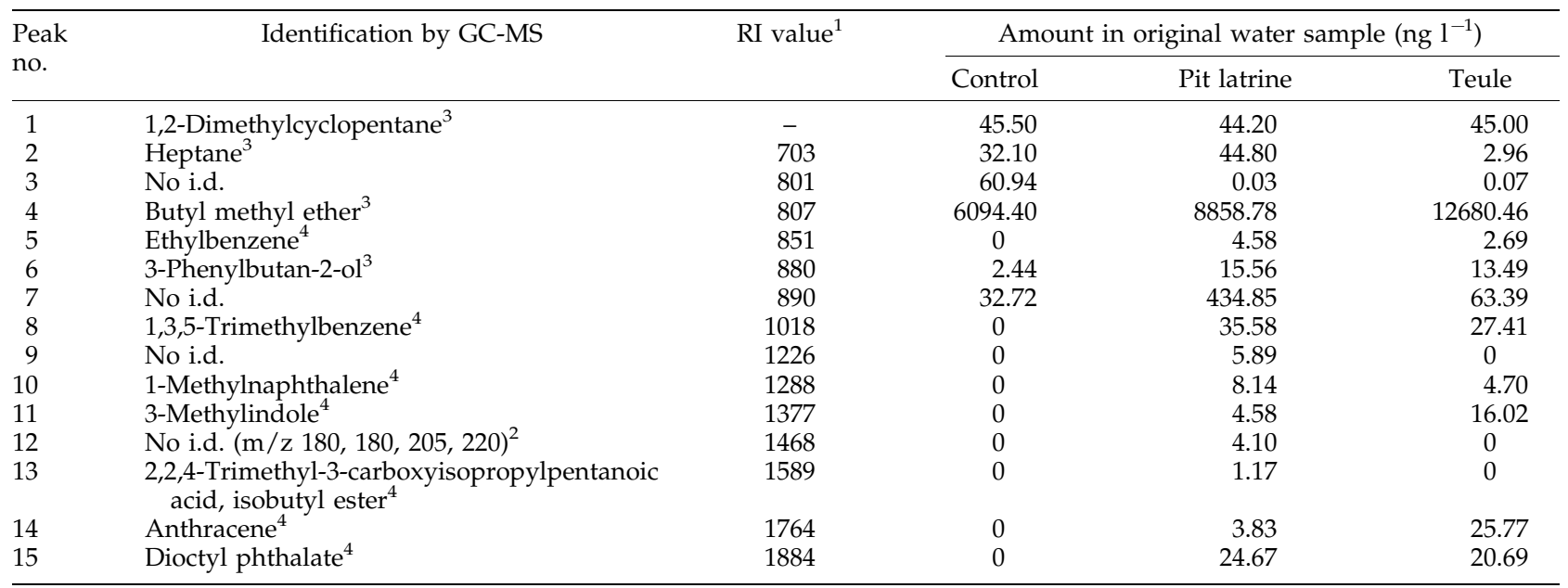

${ }^{1}$ Retention index (RI), calculated by comparison of retention times for sample fractions with n-alkanes of known molecular weight coinjected with the sample.

$2 \mathrm{~m} / \mathrm{z}=$ mass $(\mathrm{m})$ to charge $(\mathrm{z})$ ratio measurement of ions in unidentified compound.

3 tentative identification only.

4 identification confirmed by GC peak enhancement.

choice bioassays as used in this preliminary study require confirmation in future work, which should examine the effects of SBSE extracts on these behavioural responses in isolation, using techniques such as sticky screen bioassays (Isoe et al., 1995b) and wind tunnel olfactometer trials (Pile et al., 1991).

In conclusion, the SBSE technique used in this study demonstrates for the first time an effective and technically straightforward method for extraction of behaviourally active volatile fractions from $C x$. quinquefasciatus breeding site water and shows the practical feasibility of field collection of extracts for transportation and later analysis at a central laboratory facility. SBSE stir-bars will contribute to the future development of odour baits for gravid $C x$. quinquefasciatus and other mosquito species, particularly in resource-poor environments where this simple and robust field technique has significant benefits over existing methods for extraction and isolation of volatile semiochemicals. 


\section{Acknowledgements}

We thank Shahida Begum at LSHTM for providing mosquitoes used in GC-EAG work. Rothamsted Research receives grant-aided support from the Biotechnology and Biological Sciences Research Council (BBSRC) of the United Kingdom. This paper was published with permission of the Director General, National Institute for Medical Research. This paper is dedicated to the memory of Dr R.T. Rwegoshora, whose untimely death was a tragedy to his family and to those who knew him personally, and a great loss to the field of tropical entomology as a whole.

\section{References}

Baltussen, E., Sandra, P., David, F. \& Cramers, C. (1999) Stir bar sorptive extraction (SBSE), a novel extraction technique for aqueous samples: Theory and principles. Journal of Microcolumn Separations 11, 737-747.

Barbosa, R.M.R., Souto, A., Eiras, A.E. \& Regis, L. (2007) Laboratory and field evaluation of an oviposition trap for Culex quinquefasciatus (Diptera: Culicidae). Memorias do Instituto Oswaldo Cruz 102, 523-529.

Becker, N., Boase, C., Dahl, C., Kaiser, A., Lane, J., Petric, D. \& Zgomba, M. (2003) Mosquitoes and Their Control. 518 pp. New York, Kluwer Academic/Plenum Publishers.

Beehler, J.W., Millar, J.G. \& Mulla, M.S. (1994) Field evaluation of synthetic compounds mediating oviposition in Culex mosquitos (Diptera: Culicidae). Journal of Chemical Ecology 20, 281-291.

Blackwell, A., Mordue, A.J., Hansson, B.S., Wadhams, L.J. \& Pickett, J.A. (1993) A behavioural and electrophysiological study of oviposition cues for Culex quinquefasciatus. Physiological Entomology 18, 343-348.

Dethier, V.G., Browne, L.B. \& Smith, C.N. (1960) The designation of chemicals in terms of the responses they elicit from insects. Journal of Economic Entomology 53, 134136.

Du, Y.J. \& Millar, J.G. (1999) Electroantennogram and oviposition bioassay responses of Culex quinquefasciatus and Culex tarsalis (Diptera: Culicidae) to chemicals in odors from Bermuda grass infusions. Journal of Medical Entomology 36, 158-166.

Godsey Jr, M.S., Nasci, R., Savage, H.M., Aspen, S., King, R., Powers, A.M., Burkhalter, K., Colton, L., Charnetzky, D., Lasater, S., Taylor, V. \& Palmisano, C.T. (2005) West Nile virus-infected mosquitoes, Louisiana, 2002. Emerging Infectious Disease 11, 1399-1404.

Isoe, J., Beehler, J.W., Millar, J.G. \& Mulla, M.S. (1995a) Oviposition responses of Culex tarsalis and Culex quinquefasciatus to aged Bermuda grass infusions. Journal of the American Mosquito Control Association 11, 39-44.

Isoe, J., Millar, J.G. \& Beehler, J.W. (1995b) Bioassays for Culex (Diptera: Culicidae) mosquito oviposition attractants and stimulants. Journal of Medical Entomology 32, 475-483.

Kawaguchi, M., Inoue, K., Sakui, N., Ito, R., Izumi, S., Makino, T., Okanouchi, N. \& Nakazawa, H. (2004) Stir bar sorptive extraction and thermal desorption-gas chromatographymass spectrometry for the measurement of 4-nonylphenol and 4-tert-octylphenol in human biological samples. Journal of Chromatography B-Analytical Technologies in the Biomedical and Life Sciences 799, 119-125.

Kirby, W. \& Spence, W. (1826) An Introduction to Entomology. 607 pp. London, Longman, Rees, Orme, Brown, and Green.
Kramer, W.L. \& Mulla, M.S. (1979) Oviposition attractants and repellents of mosquitos - Oviposition responses of Culex (Diptera: Culicidae) mosquitos to organic infusions. Environmental Entomology 8, 1111-1117.

Laurence, B.R. \& Pickett, J.A. (1985) An oviposition attractant pheromone in Culex quinquefasciatus Say (Diptera: Culicidae). Bulletin of Entomological Research 75, 283-290.

Linstrom, P.J. \& Mallard, W.G. (Eds) (2007) NIST Chemistry WebBook, NIST Standard Reference Database Number 69, National Institute of Standards and Technology, Gaithersburg MD, http://webbook.nist.gov (accessed 20 October 2007).

Logan, J.G., Birkett, M.A., Clark, S.J., Powers, S., Seal, N.J., Wadhams, L.J., Mordue Luntz, A.J. \& Pickett, J.A. (2008) Identification of human-derived volatile chemicals that interfere with attraction of Aedes aegypti mosquitoes. Journal of Chemical Ecology 34, 308-322.

McCall, P.J. \& Eaton, G. (2001) Olfactory memory in the mosquito Culex quinquefasciatus. Medical and Veterinary Entomology 15, 197-203.

Millar, J.G., Chaney, J.D. \& Mulla, M.S. (1992) Identification of oviposition attractants for Culex quinquefasciatus from fermented Bermuda grass Infusions. Journal of the American Mosquito Control Association 8, 11-17.

Millar, J.G., Chaney, J.D., Beehler, J.W. \& Mulla, M.S. (1994) Interaction of the Culex quinquefasciatus egg raft pheromone with a natural chemical associated with oviposition sites. Journal of the American Mosquito Control Association 10, 374379.

Mordue, A.J., Blackwell, A., Hansson, B.S., Wadhams, L.J. \& Pickett, J.A. (1992) Behavioral and electrophysiological evaluation of oviposition attractants for Culex quinquefasciatus Say (Diptera: Culicidae). Experientia 48, 1109-1111.

Muturi, E.J., Mwangangi, J., Shililu, J., Muriu, S., Jacob, B., Mbogo, C.M., John, G. \& Novak, R. (2007) Evaluation of four sampling techniques for surveillance of Culex quinquefasciatus (Diptera: Culicidae) and other mosquitoes in African rice agroecosystems. Journal of Medical Entomology 44, 503-508.

Olagbemiro, T.O., Birkett, M.A., Mordue, A.J. \& Pickett, J.A. (2004) Laboratory and field responses of the mosquito, Culex quinquefasciatus, to plant-derived Culex spp. oviposition pheromone and the oviposition cue skatole. Journal of Chemical Ecology 30, 965-976.

Pile, M.M., Simmonds, M.S.J. \& Blaney, W.M. (1991) Odormediated upwind flight of Culex quinquefasciatus mosquitos elicited by a synthetic attractant. Physiological Entomology 16, 77-85.

Qiu, Y.T. (2005) Sensory and behavioural responses of the malaria mosquito Anopheles gambiae to human odours. PhD thesis, Wageningen University, The Netherlands.

Reisen, W.K. \& Meyer, R.P. (1990) Attractiveness of selected oviposition substrates for gravid Culex tarsalis and Culex quinquefasciatus in California. Journal of the American Mosquito Control Association 6, 244-250.

Reiter, P. (1983) A portable, battery-powered trap for collecting gravid Culex Mosquitos. Mosquito News 43, 496498.

Reiter, P. (1987) A revised version of the CDC gravid mosquito trap. Journal of the American Mosquito Control Association 3, 325-327.

Ritchie, S.A. (1984) Hay infusion and isopropyl alcohol-baited CDC light trap - a simple, effective trap for gravid Culex mosquitos. Mosquito News 44, 404-407. 
Rwegoshora, R.T., Pedersen, E.M., Mukoko, D.A., Meyrowitsch, D.W., Masese, N., Malecela-Lazaro, M.N., Ouma, J.H., Michael, E. \& Simonsen, P.E. (2005) Bancroftian filariasis: Patterns of vector abundance and transmission in two East African communities with different levels of endemicity. Annals of Tropical Medicine and Parasitology 99, 253-265.

Sant'ana, A.L., Roque, R.A. \& Eiras, A.E. (2006) Characteristics of grass infusions as oviposition attractants to Aedes (Stegomyia) (Diptera: Culicidae). Journal of Medical Entomology 43, 214-220.

Serodio, P. \& Nogueira, J.M.F. (2005) Development of a stirbar-sorptive extraction-liquid desorption-large-volume injection capillary gas chromatographic-mass spectrometric method for pyrethroid pesticides in water samples. Analytical and Bioanalytical Chemistry 382, 1141-1151.

Subra, R. (1981) Biology and Control of Culex pipiens quinquefasciatus Say, 1823 (Diptera, Culicidae) with Special Reference to Africa. Insect Science and Its Application 1, 319-338.

Sucharit, S., Surathin, K. \& Shrestha, S.R. (1989) Vectors of Japanese encephalitis virus (JEV): species complexes of the vectors. The Southeast Asian journal of Tropical Medicine and Public Health 20, 611-621.

Surgeoner, G.A. \& Helson, B.V. (1978) Oviposition trap for Arbovirus surveillance in Culex sp mosquitos (Diptera: Culicidae). Canadian Entomologist 110, 1049-1052. 